\title{
THE SOLAR PARTICLE EVENT OF JULY 16-19, 1966 \\ AND ITS POSSIBLE ASSOCIATION WITH A FLARE ON THE INVISIBLE SOLAR HEMISPHERE
}

\author{
HELEN W. DODSON and E. RUTH HEDEMAN \\ McMath-Hulbert Observatory, The University of Michigan, Pontiac, Mich., U.S.A. \\ and \\ STEPHEN W. KAHLER and ROBERT P. LIN \\ Space Sciences Laboratory, University of California, Berkeley, Calif., U.S.A.
}

(Received 16 July, 1968)

\begin{abstract}
An energetic solar proton and electron event was obscrved by particle detectors aboard Explorer 33 (AIMP-1) and OGO-3 during the period July 16-19, 1966. Optical and radio observations of the sun suggest that these particles were produced by a llare which may have occurred on July 16 near the central meridian of the invisible hemisphere. The active region to which the flare is assigned is known to have produced the energetic parlicle events of July 7 and 28,1966 . The propagation of the particles in the July 16-19 event over the $\sim 180^{\circ}$ extent of solar longitude from the flare to the earth is discussed, and it is concluded that there must exist a means of rapidly distributing energetic particles over a large area of the sun. Several possible mechanisms are suggested.
\end{abstract}

\section{Introduction}

From recent studies of the propagation of energetic solar particles in the interplanetary medium it has become evident that $\sim 1-30 \mathrm{MeV}$ solar protons and $\gtrsim 40 \mathrm{keV}$ solar electrons are channelled by the interplanetary magnetic field lines with essentially no propagation transverse to the field lines (O'GAL.A.AGIIER and SIMPSON, 1966; Lin et al., 1968; LIN and ANDERSON, 1967). However, energetic particles are observed from active regions on the sun which are far removed in solar longitude from the foot of the interplanetary magnetic field line connecting to the earth. Some active regions continually bathe the vicinity of the earth with encrgetic particles for a week or more, during which time the active region itsclf rotates $\gtrsim 100^{\circ}$ in solar longitude (FAN et al., 1968). In the paper of FAN et al. (1968) a model is proposed which explains the longitudinal spread of thesc energetic particles by postulating that the center of activity is directly connected by magnetic field lines in the solar atmosphere to interplanetary field lines over a $\gtrsim 100^{\circ}$ extent of solar longitude.

In this paper we shall investigate the solar particle event of 16-19 July 1966. Optical and radio observations of the sun suggest that the particles observed in this event may have been emitted by a flare occurring near the central meridian of the invisible hemisphere of the sun. Thus thesc particles apparently traversed $\sim 180^{\circ}$ in solar longitude. It is argued that the direct conncction model proposed by FAN et al. (1968) is difficult to reconcile with these observations, and several alternative means of spreading energetic solar particles over a wide area of the sun are discussed. 


\section{Particle Observations}

On July 16, 1966 an energetic solar particle event was observed by the University of California experiments on Explorer 33 and OGO-3. The OGO-3 detcctor consists of a cesium iodide crystal scintillator which records particles of energies $4<E<35 \mathrm{MeV}$ by 32 channel pulse height analysis. The Explorer 33 package consists of two thin window GM tubes and an ion chamber. One GM tube observes the particle flux scatlered off a gold foil so its directional response is limited to $>45 \mathrm{keV}$ electrons. The other GM lube is open and counts both $>22 \mathrm{keV}$ electrons and $>0.3 \mathrm{MeV}$ protons. The detectors on these satellites have been described in LIN et al. (1968).

The counting rates of the various detectors are shown in Figure 1. An increase in flux was first seen on the Explorer 33 detectors at $\sim 2230$ UT on July 16. The ion chamber shows a smooth rise to a broad maximum at 0800-1100 UT on July 17. The scatter counter shows an earlier peak at 0200-0400 UT which could be due to either (1) low energy ( $>45 \mathrm{keV}$ ) electrons or (2) protons of sufficient energy ( $\gtrsim 40 \mathrm{McV}$ ) to penetrate the shielding of the GM tube. The distinction between (1) and (2) cannot be made on the basis of the intensity-time profile of Figure 1. In order to distinguish between (1) and (2) we have obtained energic proton data from the Goddard Space Flight Center $E \cdot v s$. $\mathrm{d} E / \mathrm{d} x$ experiment on IMP-3 (MCDonal.d, 1968). Those data are organized in 6-hour intervals. The maximum proton flux of the 38 to $59 \mathrm{MeV}$

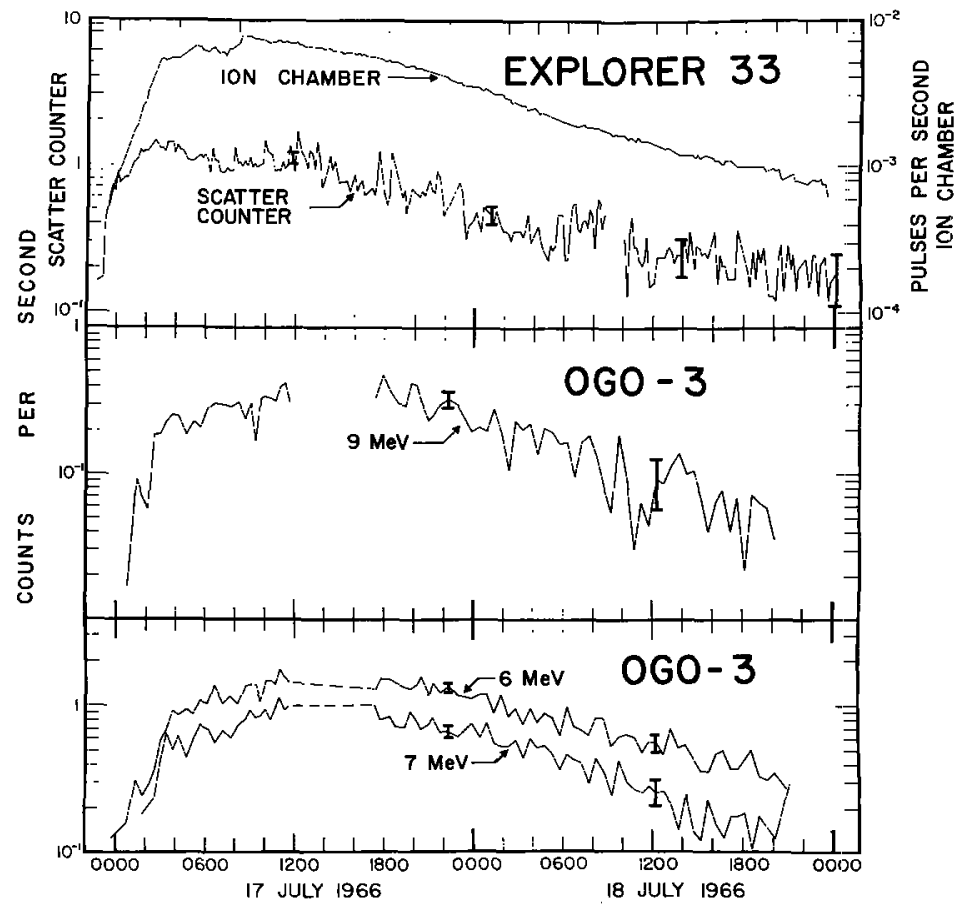

Fig. 1. Counting rates of the Explorer 33 ion chamber and scatter counter and three of the OGO-3 channels. In the OGO-3 detector, 1.7 counts $/ \mathrm{sec}-1$ particle $/ \mathrm{cm}^{2} \mathrm{sec} \mathrm{sr} \mathrm{MeV}$. 
interval occurred during the first 6 hours of July 17 and was 0.02 particles per $\mathrm{cm}^{2} \mathrm{sr} \mathrm{sec}$. The $59-80 \mathrm{MeV}$ interval had a counting rate in the same period of 0.008 particles per $\mathrm{cm}^{2} \mathrm{sr} \mathrm{sec}$. The contribution to the scatter counter count rate from penetrating protons is estimated from these figures to be $\leqslant 0.3$ counts per second. This is well below the observed increase of 1.6 counts per second. Therefore, most of the increase in the scatter counter must be due to electrons $>45 \mathrm{keV}$. During the time of the observations reported here, Explorer 33 was located just outside the geomagnetic tail on the dawn side at geocentric distances greater than $55 R_{\mathrm{c}}$. The OGO-3 orbit has an apogee of $\sim 20 R_{\mathrm{e}}$ and the sun-earth-apogec angle projected onto the ecliptic plane of $123^{\prime \prime}$ (measured toward dawn). No data is shown during the time when OGO-3 is within the region of trapped radiation.

\section{Associated Solar Phenomena}

Lists of worldwide flare observations (IAU Quarterly Bulletin on Solar Activity, 1966 and ESSA Bulletin, 1966) provide no indication of the occurrence of a suitable flare as the source of these particles. This situation makes necessary a special study of the optical solar records for the days and hours prior to the onset of the particle event.

First it is necessary to examine the statement that there was no appropriate flare as the source of the high-energy particles. This statement does not mean that subflares did not occur during the preceding days and hours. Several subflares did occur and were reported to World Data Centers. The statement does mean that reexamination of $H x$ records shows that the subflares during the preceding ten hours were truly minor solar phenomena and were not accompanied by either ionospheric disturbances or enhancements at radio frequencies (single frequency records). In the past, ccrtain electron or proton events have been ascribed to solar subflares. In these cascs reexamination of the data generally has indicaled that the subflares in question werc solar events of some significance with accompanying radio frequency or ionospheric phenomena. According to all data currently available, the subflares reported from 1200 to 2240 LT on July 16 , referred to only minor solar phenomena.

In addition, a recent reexamination of the flare data in the Quarterly Bulletins of Solar Activity for 1964-1966 on the basis of evidence from all actually patrolling stations, indicates that the last flare prior to the proton-electron event of July 16 for which the majority of observers concurred in an importance evaluation $\geqslant 1$ took place on July 11 . The interval July 12 to 20 was without any confirmed flares with importance as great as $\mathrm{l}$. The $\mathrm{H} \approx$ flare patrol was unbroken from July $13-16$ so an argument for a 'missed flare' during the critical interval cannot be supported. The abscnce of reports of ionospheric disturbances of all kinds July 14-16 attests further to the lack of major flares during the days and hours immediately prior to the electron-proton event of July 16-19, 1966.

On the other hand, examination of the $\mathrm{H} x$ records of the McMath-Hulberi Observatory for July 16, 1966 reveals the occurrence of an interesting and major solar event approximately two hours before the first detection of solar electrons and 
protons. An eruplive prominence was observed from 2051 to $\sim 2120$ UT on the Northwest limb of the sun at the very high latitude of $70^{\circ}$ (see Figure $2 \mathrm{c}$ ). The prominence extended at least $80000 \mathrm{~km}$ beyond the limb and was visible most readily on records made with radiation $1 \AA$ or more from the center of $\mathrm{H} x$. The observations do not permit a determination of outward velocity.
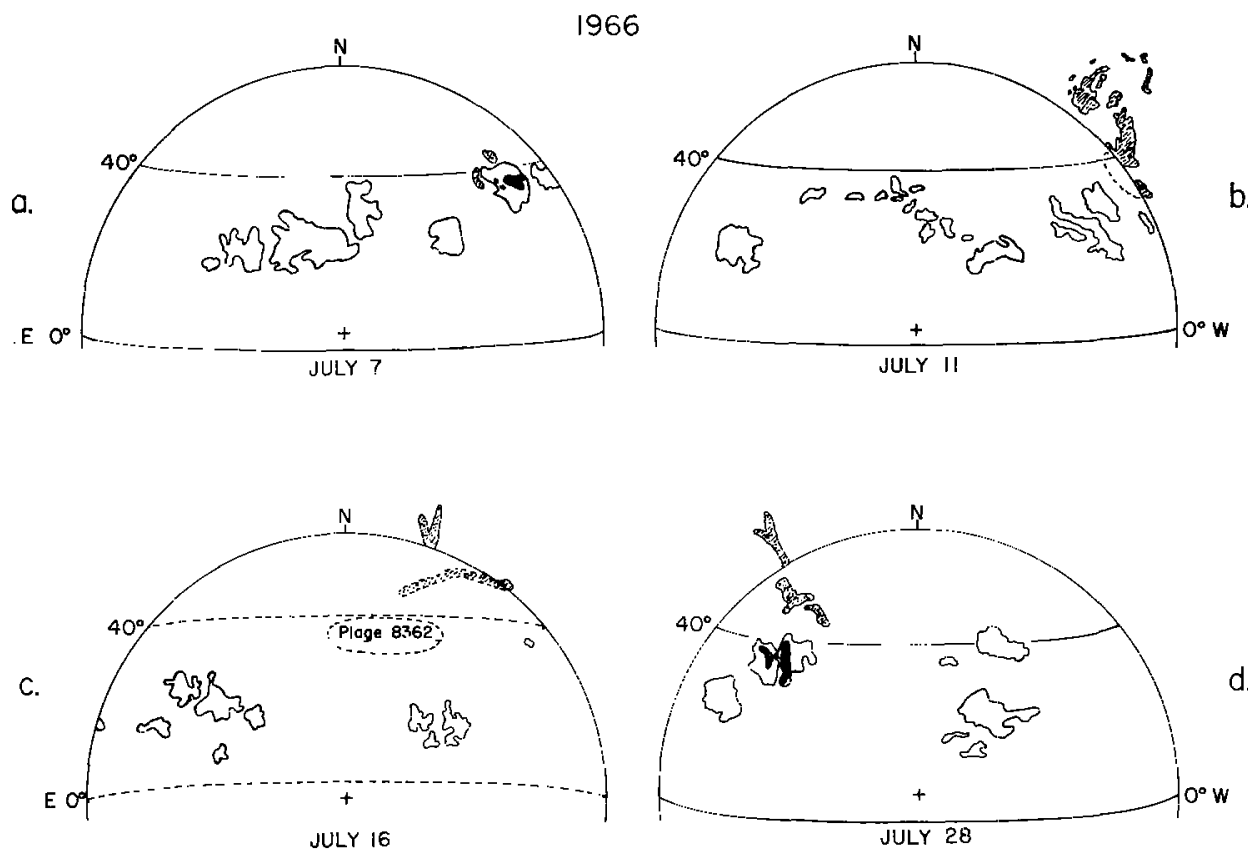

Fig. 2. Diagrams showing flares, filaments and eruptive promincnces in the neighborhood of the center of activity of the proton-clectron flare of July 7, 1966. Dotted lincs refer to featurcs inferred to be on the invisible hemisphere. Plages have been traced from l-raunhofer Iristitute Maps. Flares are shown in black; prominences and fjlaments are shaded. Filaments and prominences not near the center of activity under study are omitted from the diagrams. (a) July 7: Flare, 0048 UT; Lockheed film; (b) July 11: Eruptive prominence, 0950 UT, copied from Fraunhofer Institute Map; (c) July 16: Eruptive prominence $\sim 2100 \mathrm{UT}$, McMath-Hulbert film; (d) July 28: Flare and cruptive prominence $\sim 2315$ UT, McMath-Hulbert film.

The eruptive prominence referred to here was not an instance of a previously visible, quiescent prominence becoming active and eruptive. Nor was it a case in which a filament on the disk became active, approached the limb, and then appeared as an eruptive prominence. Therefore, one is led to associate the eruptive prominence of July 16, 1966 with aclivity taking place on the invisible hemisphere.

Some, but not all, eruptive prominences are known to occur concomitantly with major flares. Great prominence ejections frequently occur in the premaximum phase of flares (Donson et al., 1953). They also may occur later in the devclopment of the flare and in these cases may be associated with the onset of activity in a previously existing, neighboring filament (DODSON and HEDEMAx, 1968a). Accordingly, one can 
argue that the occurrence of the eruptive prominence on July 16, 1966 implies the possible existence of a major flare on the invisible hemisphere.

Radio frequency data provide limited evidence in support of the occurrence of a flare at $\sim 2051 \mathrm{UT}$ on the invisible hemisphere. There is no report of enhancement at the high radio frequencies which normally are associated with disturbances low in the solar atmosphere. If they occurred in the invisible hemisphere of the sun they would be occulted by the solar disk. From 2120 to 2200 UT on July 16, the Boulder station reports weak continuum emission, 41-24 MHz. This was followed by a welldefined, distinct burst, importance $1+$ or 2 , at $18 \mathrm{MHz}, 2207-2214 \mathrm{UT}$ on the records of three stations. Finally, Fort Davis reported a minor type I noise storm, $280-100 \mathrm{MHz}$ from 2247 to $2300 \mathrm{UT}$. The time sequence of events is summarized in Table I.

TABLE I

Sequence of Events on July 16, 1966

Eruptive prominence visible, $\mathrm{N} 70^{\circ}$, west $\operatorname{limb}$
Continuum emission, Imp. $1,41-24 \mathrm{MHz}$
Burst, Imp. $1+$ or $2,18 \mathrm{MHz}$
Type I noise storm, Intensity $1,280-100 \mathrm{MHz}$
Start of proton event, $E>15 \mathrm{MeV}$, Explorer 33
Start of electron event, $\gtrsim 40 \mathrm{keV}$ electrons, Explorer 33

It is important to recall that on July 16, 1966 a center of activity, McMath Plage 8362 , with a previous history of proton-electron emission was on the invisible hemisphere of the sun. On July 16, this region was approaching the central meridian of the invisible hemisphere $\left(\sim 8^{\circ}\right.$ to the West). Although close to the central meridian, the region was not close to the center of the invisible disk. Its high latitude, combined with the tilt of the sun's axis of rotation, put the region $\sim 40^{\circ}$ from the center of the invisible disk. In this position, radially outward ejections from the region would be visible to the earth at all heights above the photosphere greater than $\sim 0.6$ of a solar radius. We suggest that the small proton-electron event of July 16-19, 1966, observed near the earth may have resulted from an ejection of particles from a flare in this center of activity on the invisible hemisphere.

There is one further piece of circumstantial evidence to support the above suggestion. Observations show that major flares in the center of activity in question tended to be accompanied by eruptive prominences at very high latitude. ÖHMAN et al. (1967) has reported a major flare and great eruptive prominence on 0904 UT July 11, 1966, when Region 8362 was one day beyond the West limb (see Figure 2b). There is no report of protons or electrons with this flare. Again, on 2216 UT July 28, 1966 when the same region was $33^{\circ}$ East of the visible central meridian in its second transit across the solar disk, another major flare occurred within its boundaries (DoDson and Hedeman, 1968). This flare was accompanied by the apparent disintegration of a filament to the North and East $\left(\mathrm{N} 50-60^{\circ}\right)$ and by the appearance of a major eruptive prominence at $\mathrm{N} 62^{\circ}$ at $2310 \mathrm{UT}$ (see Figure $2 \mathrm{~d}$ ). Low energy protons $(E>0.5 \mathrm{MeV}$ ) 
and electrons $(E \gtrsim 40 \mathrm{keV})$ were delected by IMP-3 before 1700 UT on July 29 . The particle event increased slowly to a maximum on July 31.

An event similar to the eruptive prominence of July 28, 1966 may have occurred on July 16,1966 . The existence of a neighboring 'filament' at $\sim 50-60$ "N latitude on this date on the invisible hemisphere can be deduced from observation of prominences at these latitudes on the West limb, July 13-16. Accordingly the high latitude eruptive prominence of July 16, 1966 indicates activity occurring primarily in the invisible solar hemisphere, and provides indirect evidence for another major flare in the center of activity that was the site of the electron-proton flares of July 7 and 28, 1966.

\section{Time of Particle Injection}

One of the earliest models to account for the intensity-time profiles of solar proton events was the model of isotropic diffusion of the particles in interplanetary space (PARKER, 1963). In this model the parlicle flux at a given energy varies with time as $I=1 / t^{3 / 2} \exp \left[-3 t_{\mathrm{m}} / 2 t\right]$ ( $I=$ flux, $t=$ time after injection and $t_{\mathrm{m}}$ is the time of maximum flux observed at the earth) so that a plot of $\log I t^{3 / 2}$ versus $1 / t$ should give a straight line whose slope $S$ is given by $S=(3 / 2) t_{\mathrm{m}}$. This value of $t_{\mathrm{m}}$ is thus derived in two different ways and serves as a check on the validity of the fit.

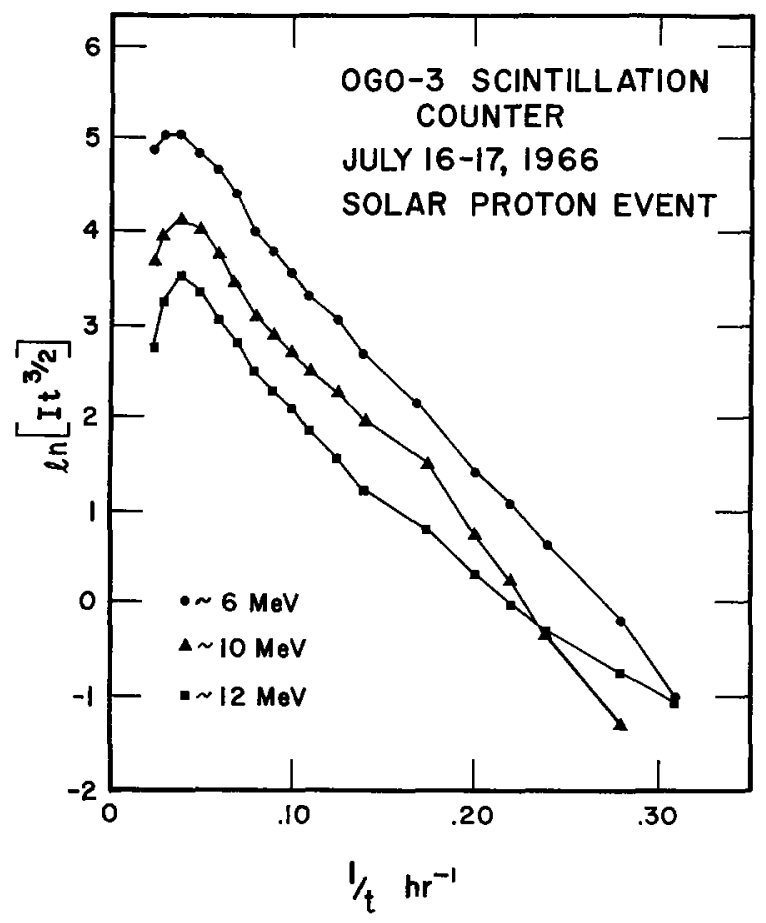

Fig. 3a.

Fig. 3. (a) lsotropic diffusion model fits for the counting rates of three OGO-3 channels; (b) Same as (a) for the Fxplorer 33 ion chamber. $t$ is time after 2100 UT on July 16. 


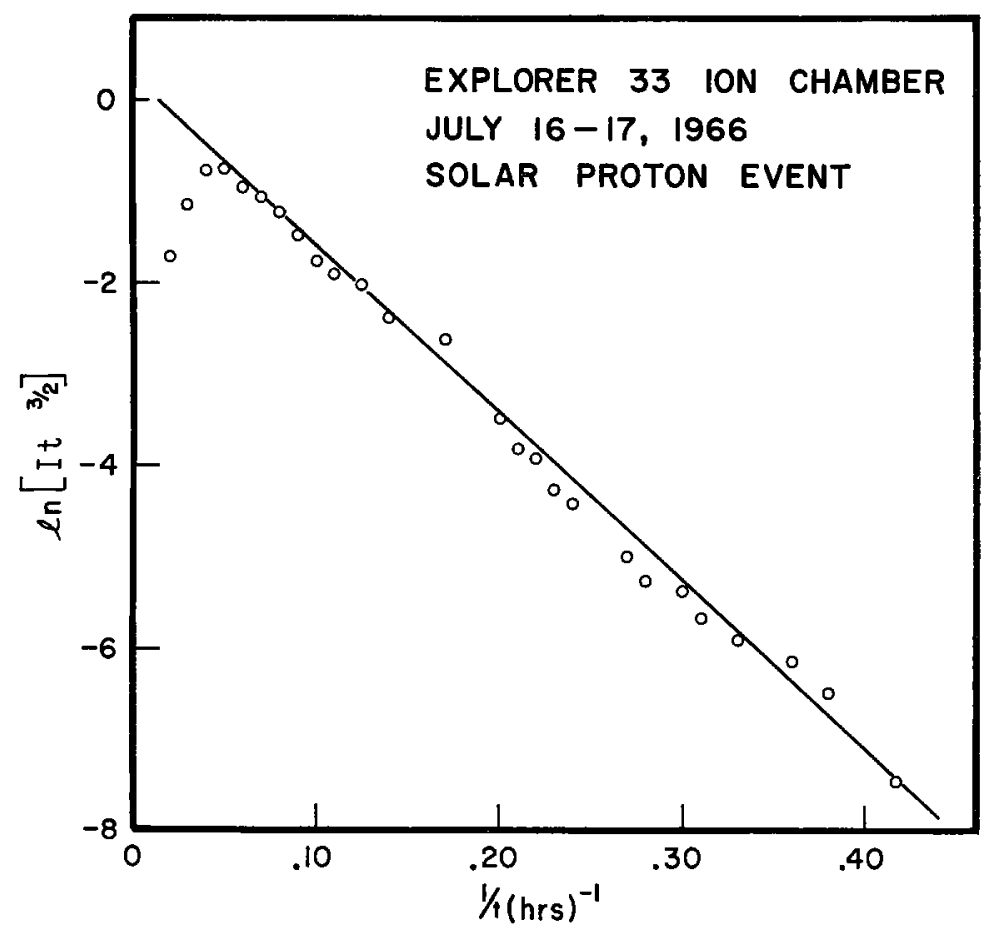

Fig. 3b.

We have taken as the starting lime 2100 UT on July 16 at which time the eruptive prominence described in Section 2 was seen. Figures $3 \mathrm{a}$ and $\mathrm{b}$ show the fits of the ion chamber and several channels of the OGO detector to the diffusion model. The OGO data is subject to large statistical errors due to the low particle flux and the high background count rate of the OGO detector. The Explorer 33 ion chamber because of its large geometry factor has excellent statistical accuracy, but its response is dependent on the spcctrum of the particles. During this event, the spectrum of the protons as observed by the OGO-3 detector does not vary substantially, so that the time history of the flux observed by the Explorer 33 ion chamber is indicative of the time history of flux at one encrgy. For the ion chamber the slope was measured to be 18.4 hours which gives $t_{\mathrm{m}} \sim 12.3$ hours. This corresponds to $0915 \mathrm{UT}$ on July 17 ; the observed flux maximum was at 0927 UT.

Our purpose in discussing the isotropic diffusion model for this particle event has not been to argue a case for isotropic diffusion. Theoretical (JoKIPII, 1967; Ror:Lor, 1966) and experimental (MCCRACKEN et al., 1967; LIx et al., 1968) studies have shown conclusively that the isotropic diffusion model is not valid. Our purpose in presenting these fits is to show that this event is similar to other prompt flare-initiated proton events and, most important, that an injection time of $2100 \mathrm{UT}$, corresponding to the time of the eruption of the solar prominence, gives a diffusion model fit in 
which the values of $t_{\mathrm{m}}$ derived from the slope of the fit and from the time of the peak counting rates are in good agreement. Changes of $\gtrsim 30 \mathrm{~min}$ in the injection time will lead to much poorer fit to a straight line. In the summary by WEBBER (1964) the starting times derived by this method for the events studied were always very close to the time of the initiating flare at the sun.

\section{Discussion}

We have presented evidence in the preceding discussion that the solar particle cvent of July 16-19, 1966 probably was associated with a solar flare in calcium plage region 8362 , the well-established source of solar protons on July 7 and 28 . Since this center of activity was near central meridian of the invisible hemisphere of the sun on July 16 , the event presents an interesting case of coronal and interplanetary particle propagation.

FAx et al. (1968) have proposed a model for the origin and propagation of longlived proton fluxes observed on Pioneer 6 and Pionecr 7. They associatcd the observed enhancements of $0.6-13 \mathrm{MeV}$ proton fluxes with specific active regions on the sun. These enhanced proton fluxes were present for time intervals during which the associated active regions rotated through as much as $\sim 180^{\circ}$. During these times of continuously cnhanced $0.6-13 \mathrm{MeV}$ proton fluxes, discrete proton events with rapid transit times and onsets were observed. These events were clearly associated with flares in the active region. These obscrvations led the authors to a model in which the magnetic field lines from an active center are connected to interplanctary field lines in the solar corona over a longitude range of $100^{\circ}-180^{\circ}$. Discrete proton cvents seen at the earth are considered to be the result of particles traveling from the flare to the earth by directly connecting field lines rather than by a diffusion process since diffusion over a wide range of longitude would lead to much slower onsets.

It should be noted that optical studies (DODSox and Hrideman, 1968b and 1968c) have led to a concept of 'zones' of activity on the sun much larger than single plages. The zones of activity persist for long intervals of lime (scveral years) and extend across $\sim 70^{\circ}-90^{\circ}$ longitude.

Calcium plage, number 8362 , in which the flare on the invisible hemisphere on July 16 may have occurred, is one of the regions which FAN et al. (1968) associate with enhanced proton fluxes. An enhanced 0.6-13 MeV proton flux was first observed on July 4 at which time the region was at $\sim 5^{\circ}$ West of the central meridian. The enhanced proton flux cut-off for this region was reported to have occurred after July 14. Extension of the cul-off date to include the proton event of July 16-19 lcads to geometrical circumstances that appear unreasonable for their model. The high. latilude of region 8362, and the inclination of the sun's axis of rotation, combine to put the region $\sim 40^{\circ}$ from the center of the invisible hemisphere on July 16 . If the lines of force from an active region spread out over a broad interplanetary region as suggested by F $\Lambda \mathrm{N}$ et al. (1968) then there is a possibility that the lines from region 8362 extended substantially over the Northern polar cap of the sun. This possibility 
seems unlikely in the light of polar plume observations which indicate that the magnetic field over the pole is essentially radial.

If the July 16-19 proton event did not have its source in plage 8362 then Table 3 of the paper by FAN et al. (1968) suggests region 8379, four days to the East of 8362 as another signilicant center of solar activity. On July 16 this region was also on the invisible hemisphere, about two days beyond transit of the sun's West limb. We know of no observations that suggest a positive association of the July 16-19 proton event with region 8379 . It is possible that region 8379 , along with 8362 , formed part of a single large 'zone' of solar activity extending over $\sim 60^{\circ}$ of longitude that included not only the July 7 proton region but also the antecedents of the August 28-Scptember 2 proton center.

To summarize, the interpretation of the July 16, 1966 solar particle event according to the model of FAv et al. (1968) requires that plage 8362 plus its important neighbors be magnetically connected to the earth even though that region is near central meridian of the invisible hemisphere. This appears to be an untenable requirement in view of studies of the solar magnetic field through indirect means such as solar plumes, and directly through studies of the interplanetary sector structure (WILCOX and Ness, 1965) and its evolution (ScitatTen et al., 1968). Such studies of the relationship of the interplanetary field to the solar photospheric field show that the interplanetary field is best correlated to the large unipolar magnetic regions which have relatively weak fields rather than to the magnetic fields of active regions.

The presence of $\geqslant 45 \mathrm{keV}$ electrons makes it difficult to invoke scattering by spatial magnetic irregularities for particle propagation across ficld lines. In a coronal field of $\sim 1$ gauss, the gyroradius of a $\sim 45 \mathrm{keV}$ electron is $\sim 10^{-3}$ times the gyroradius of a $10 \mathrm{MeV}$ proton. Thus the electrons would presumably diffuse across magnetic field lines more slowly than the protons; but the electron fluxes observed in the scatter counter of Explorer 33 on July 16 showed a much sharper onset and carlier peak than the proton fluxes.

There are several possible means of spreading particles over a large region in the solar corona to reach interplanelary field lines connecting to the earth. First the particles may be accelerated over a large region of the corona. This acceleration can be accomplished by means of shock waves traveling through the solar corona such as those reported by ATHAY and Morizron (1961) and SMITH and ANGi.e (1968). Electrons may be easily accelerated to $\gtrsim 40 \mathrm{keV}$ by shock waves, but protons, because of their high rigidity, are less likely to be accelerated. Studies by LIN and ANDERSON (1967) indicate that electrons might very well be accelerated separately from protons. Another possibility is the rapid transport of these particles across coronal magnetic field lines by means of hydromagnetic wave disturbances. Hydromagnetic waves with wavelengths of the order of these particles' gyroradii would allow the particles to violate the first adiabatic invariant and travel across field lines rapidly.

Large-scale structures such as filaments and prominences suggest the possibility of mass transport of energetic particles along field lines spatially ordered over large 
distances. In such a process, diffusion is required only from the filament or prominence to the presumably adjacent interplanetary field lines.

Further study of the relation of energetic particle emission to optical and radio phenomena at the sun are needed to choose between these possibilities.

\section{Acknowledgments}

One of us (S.W.K.) wishes to acknowledge helpful discussions with Dr. J. M. Wilcox. This research was sponsored in part by NASA contracts NAS 5-9077, N $\Lambda$ S 5-2222 and NASA-NGR-23-005-275.

\section{References}

Athay, R. G. and Moreton, G. E.: 1961, Astrophys. J. 133, 935.

Dodson, Helen W., Hedeman, E. Ruth, and Chamberi ain, J.: 1953, Astrophys. J. 117, 66.

Dodsov, Helen W. and Hedeman, F. Ruth: 1968a, Report of 1966 July 7 proton flare project: Late development of center of activity, Annals of IQSY, MIT Press, Cambridge, Mass. (in press).

Dodson, Helen W. and Hfoeman, E. Ruth: 1968b, ITistory and morphology of solar activity, 1964-65, Annals of IQSY 4, MIT Press, Cambridge, Mass. (in press).

Donson, Helen W. and Hedeman, F. Ruth: 1968c, 'Some patterns in the development of centers of activity, 1962-1966', in IAU Symposium No. 35 (edited by K.O. Kiepenheuer), p. 56.

Fan, C. Y., Pick, M., Pyi.e, R., Simpson, J. A., and SmIn, D. R.: 1968, f. Geophys. Res. 73, $1555-1582$.

JokJPII, J. R.: 1967, Astrophys. J. 149, 405.

Lin, R. P. and ANDirson, K. A.: 1967, Solar Phys. 1, 446-464.

Lin, R. P., Kattiter, S. W., and Roelof, E. C.: 1968, Solar Phys, 4, 338.

McCracken, K. G., Rao, L. R., and BukniA, R. P.: 1967, J. Geophys. Res. 72, 4293.

MCDONAI.J, F.B. : 1968, private communication.

O'Gnllagher, J. J. and Simpson, J. A.: 1966, Phys. Rev. Letters 16, 1212-1217.

Öhmav, Y., Strlber, G., and Kusorfsky, U.: 1967, Solar Phys. 1, 60.

PARKer, E. N.: 1963, Interplanetary Dynamical Processes, Intcrscience Publishers, New York.

RoElof, E. C.: 1966, Stalistical theory of charged particle transport in disordered magnetic fields, Ph.D. Dissertation, University of California, Physics Department, Berkeley.

Schatten, K. M., Wilcox, J. M. and Ness, N. F.: 1968, Inlerplanctary magne.ic ficld structure and scctor evolution, presented to special meeting of the American Astronomical Socicly, Tucson, Arizona.

SmITH, S. H. and ANGLE, K. I.: 1968, Observational ellects of flare-associated high speed "waves', presented at Midwest Cosmic Ray Conference, University of lowa.

Solar-Geophysical Data, Environmental Science Scrvices Administration/Institute for Telecommunication Science and Aeronomy, Washington D.C.: 1968.

Webrer, W. R.: 1964, in AAS-NASA Symposium on the Physics of Solar Flares, NASA SP-50, Washington, D.C.

WII.Cox, J. M. and Ness, N. F.: 1965, J. Geophys. Res. 70, 5793. 